\title{
Long-term survival after endovascular and open repair of unruptured
}

abdominal aortic aneurysms in England between 2006 and 2015: a retrospective cohort study

A S Johal1, I M Loftus2, J R Boyle3, K Heikkila1,4, S Watonı, D A Cromwell1,4 Type of Study: Original Article

Amundeep S Johal, PhD

Ian M Loftus, MD

Jonathan R Boyle, MD

Katriina Heikkila, $\mathrm{PhD}$

Sam Waton, BSc

David A Cromwell, PhD

1 The Clinical Effectiveness Unit, The Royal College of Surgeons of England, 35-43 Lincoln's Inn Fields, London, UK WC2A 3PE

2 St George's University Hospitals NHS Foundation Trust, Blackshaw Road, Tooting London, UK SW17 0QT

3 Division of Vascular and Endovascular Surgery, Addenbrooke's Hospital, Cambridge University Hospital Trust, Cambridge, CB2 0QQ

${ }_{4}$ Department of Health Services Research \& Policy, London School of Hygiene \& Tropical Medicine, Keppel Street, London WC1E 7HT

Address to which correspondence should be addressed:

Dr A S Johal

The Clinical Effectiveness Unit, The Royal College of Surgeons of England, 35-43 Lincoln's Inn Fields, London, UK WC2A 3PE Email: ajohal@rcseng.ac.uk, Tel: (+) 442078696628 


\section{Sources of Funding}

This study was conducted as part of the National Vascular Registry, which is commissioned by the Healthcare Quality Improvement Partnership (HQIP) as part of the National Clinical Audit (NCA) Programme. HQIP is led by a consortium of the Academy of Medical Royal Colleges, the Royal College of Nursing and National Voices. Its aim is to promote quality improvement, and in particular, to increase the impact that clinical audit has on healthcare quality in England and Wales. HQIP holds the contract to manage and develop the NCA Programme, comprising more than 30 clinical audits that cover care provided to people with a wide range of medical, surgical and mental health conditions. The programme is funded by NHS England, the Welsh Government and, with some individual audits, also by the Health Department of the Scottish Government, Department of Health, Social Services and Public Safety of Northern Ireland and the Channel Islands. This publication is based on data collected by or on behalf of HQIP, which has no responsibility or liability for the accuracy, currency, reliability and/or correctness of this publication.

\section{Supporting information}

Online Appendix. Supplementary tables and figures

\section{Conflicts of Interest}

Prof I Loftus declares a consultancy agreement with Endologix, Irvine California, USA. Prof I Loftus also declares grants from Medtronic Ltd, Watford, UK. The remaining authors have no conflicts to disclose.

\section{Ethics approval}

The study is exempt from UK National Research Ethics Committee approval as it involved secondary analysis of an existing dataset of anonymised data. HES data were made available by the NHS Digital (Copyright $\odot$ 2015, reused with the permission of NHS Digital. All rights reserved). 


\section{Data sharing}

The authors do not have permission to share patient-level HES data. HES data are available from the NHS Digital Data Access Advisory Group (enquiries@nhsdigital.nhs.uk) for studies who meet the criteria for access to confidential data.

\section{Preregistration of study}

No preregistration exists for the analyses reported in this article. 


\section{ABSTRACT}

Background: The aim of this paper was to examine patterns of 10-year survival after elective repair of unruptured abdominal aortic aneurysms (AAA) in different patient groups.

Methods: Patients having open repair (OR) or endovascular aneurysm repair (EVAR) in the English NHS between January 2006 and December 2015 identified from Hospital Episode Statistics data. Postoperative survival among patients of different age and Charlson comorbidity profiles were analysed using flexible parametric survival models. The relationship between patient characteristics and risk of rupture after repair was also analysed.

Results: 37,138 patients underwent elective AAA repairs, of which 15,523 were open and 21,615 were endovascular. 10-year mortality was $38 \%$ for patients under 70 years, and the survival trajectories for OR and EVAR were similar when patients had no RCS comorbidity. Among older patients or those with comorbidity, 10 -year mortality rose exceeding $70 \%$ for patients aged 80 plus. Mean survival times over 10-years for OR and EVAR were often similar within subgroups of older and more comorbid patients but their survival trajectories became increasingly dissimilar, with OR showing greater short-term risk within 6-months but lower 10-year mortality rates. The risk of rupture over 9-years was $3.4 \%$ for EVAR and $0.9 \%$ for open repair, being weakly associated with patient factors.

Conclusion: Long-term survival patterns after elective OR and EVAR for unruptured AAA vary markedly across patients with different age and comorbidity profiles. Judgements about the most appropriate technique require shared decision making so patients can balance the short-term and longterm risks.

Keywords: Abdominal aortic aneurysm, endovascular, long-term survival, flexible parametric regression models

(Abstract $=245$ words; Main text $=\mathbf{3 5 1 2}$ words $)$ 


\section{INTRODUCTION}

Endovascular repair (EVAR) has become the most common technique for elective repair of abdominal aortic aneurysms (AAA) in the UK. In 2007, according to the Hospital Episode Statistics database (HES), EVAR procedures accounted for $36 \%$ of all first time elective AAA repairs in English NHS hospitals, but this had increased to $74 \%$ in 2015. Studies on its relative effectiveness compared with open repair (OR) have typically found EVAR to have a lower short-term postoperative mortality, with a recent systematic review estimating 30 -day/in-hospital mortality to be $1 \cdot 3 \%$ compared with $4.7 \%$ for $\mathrm{OR}_{1}$. However, long-term follow-up of patients in the randomised controlled trials (RCT) comparing EVAR with OR have found the early survival benefit of EVAR is not sustained2-5. EVARs are susceptible to device failure, endoleaks, migration and other complications that require further intervention or surgery and, consequently, there is a need for long term surveillance2,4.

The long-term outcomes of elective EVAR and OR have also been examined by various populationbased cohort studies6-9. These benefited from having less restrictive eligibility criteria compared to the trials, which in general had patients with fewer comorbidities than the general AAA popualtion 10,11, but they have produced inconsistent results. Compared with OR, cohort studies have reported EVAR

to have lower all-cause mortality after 5 years7, or have a higher 4-year mortality rates,9, while another found that survival beyond 3-years was similar for EVAR and OR6. Why these differences have arisen is not clear. Possible reasons include the different settings and study time periods as well as variation in the degree of heterogeneity within the patient cohorts.

The influence of patient factors such as age and comorbidities (like congestive heart failure, respiratory disease and renal impairment) on long-term survival after AAA repair has been well documented 12 and the large, representative samples of patients in the cohort studies allow for the effect of different patient factors to be evaluated. However, the population-based cohort studies have 
tended to focus on determining the comparative effective of EVAR and OR, using either a standard Cox regression 8,9 or a method based on propensity scores6,7. Little attention has so far been given to how the patterns of short and long-term mortality might differ among patient groups, and in what circumstances the hazard ratio of EVAR to OR changes. That the survival curves for EVAR and OR cross after several years suggests the hazard ratio may not be constant and the proportional hazards assumption required for standard Cox regression is not valid. Schermerhorn et al6 was one exception, using piecewise Cox regression to estimate the hazards at 30-days, 90-days, and 4 years postintervention, and noted the early advantage of EVAR increased among older patients.

The aim of this study was to examine the long-term risks of death and rupture for unruptured elective OR or EVAR in a population-based cohort. These outcomes were investigated for different patient groups (defined in relation to patient age and the presence of specified comorbidities) using an analytical approach which allowed for the ratio of the mortality rates after EVAR and OR to change over time 13,14

\section{METHODS}

\section{Patient population and exclusions}

The study used data extracted from the HES database and identified patients admitted for elective AAA repair between 1 January 2006 and 31 December 2015. HES records code diagnostic information using the International Classification of Diseases, 10th revision (ICD-10), and operative procedures using the UK Office for Population Censuses and Surveys classification (OPCS), 4th revision. The study cohort contained patients admitted electively with a diagnosis of unruptured AAA (ICD-10 code: I71.4) and that had either a first time (index record) OR procedure (L19.4, L19.5, L19.6, L19.8) or an EVAR (L27·1, L27·5-9 or L28·1, L28·5-9). The index record was defined as the first hospital admission in which a patient had AAA repair. The few index records that described patients having both types of procedures ( 241 records) were removed. The few records that coded the 
procedure as OR but which also contained codes for the use of a stent graft were re-defined as an EVAR procedure.

\section{Study variables}

Demographic variables for age and sex were obtained from the index procedure record. The presence of comorbidity was described using the Royal College of Surgeons of England's modified Charlson score15. This score is based on 14 acute and chronic conditions that are typically associated with survival after surgery, many of which have been linked with worse outcomes in patients who undergo AAA repair12 such as: myocardial infarction, congestive heart failure, chronic pulmonary disease, renal impairment, cerebrovascular disease, diabetes and peripheral vascular disease. The score counts the number of times each chronic condition is recorded in the index admission or is present in HES records 12 months prior to this; acute conditions (for example acute myocardial infarction) are only counted among records from 12 months prior to the index admission but not the index admission itself. The score does not include hypertension or cardiac arrhythmias (atrial fibrillation), and patients with a RCS Charlson score of 0 may suffer from some degree of comorbidity.

The primary outcome was postoperative all-cause survival, which was defined as the time from surgery to the date of death. The date and cause of death was obtained from the Office for National Statistics death register, which provided complete follow-up. However, deaths were rarely attributed to abdominal aneurysms beyond the postoperative period and cause of death was therefore uninformative about long-term risks and is not reported. The last date of death available was 16 October 2016, which gave a minimum follow-up time of 290 days. The mean time of follow-up was 4. 4 years. The secondary outcome was time from surgery to any readmission for a ruptured aneurysm (defined as ICD-10: I71·1, I71·3, I71.5 \& I71·8).

\section{Statistical Analysis}

The pattern of long-term survival among patients having EVAR and open repairs was examined in two steps. The association of survival with age, sex and the RCS comorbidity score was initially 
evaluated using Kaplan-Meier survival curves and a standard Cox regression model. Cox regression was also used to examine the association of time-to-rupture with these patient characteristics. Log-log plots and the Schoenfeld residual test were used to assess whether the survival and time-to-rupture data followed the proportional hazards assumption of the Cox regression model 16 . This assumption proved acceptable for time-to-rupture but not for survival. In the latter case, the hazard ratio of EVAR compared with OR was found to change over time.

In the second step, the results of the initial Cox model for survival were used to partition the cohort into patient groups that distinguished between patient age and presence of comorbidities. The patterns of long-term survival within each patient group were analysed using a flexible parametric regression model 13 with procedure type included as a time-varying variable. This avoided the simplification associated with piecewise Cox regression, namely, that the hazards are assumed to be fixed over predefined intervals (the resulting step-functions are rarely plausible biologically13). In flexible parametric regression, a restricted cubic spline is used to model the hazard function of each procedure as a continuous variable. The appropriate spline was selected using the Akaike Information Criterion and Bayesian Information Criterion (measures of how well a statistical model fits the data13) and a visual inspection of the hazard functions to avoid overfitting the model.

Because the hazard ratio of EVAR compared with OR was not constant, the differences in estimated survival times of the two procedure types are summarised using the restricted mean survival time (RMST) 14 for the 10-year follow-up period. This gives the expected survival time for patients having either procedure for the specified follow-up period and is a common method for comparing survival when the hazards are not proportional. Statistical calculations were performed with Stata version $15 \cdot 1$ MP, (Stata Statistical Software, College Station, TX, USA, www.stata.com). 


\section{RESULTS}

There were 37138 patients who were admitted for an elective repair of AAA between 1 January 2006 and 31 December 2015 . Only $12 \cdot 2 \%$ of patients were female (Table 1 ), and the patients who had EVAR tended to be older (with a mean age of 75.5 years compared to 71.9 years for OR patients) and more inclined to have one or more Charlson comorbidities (68.3\% compared to 59.6\% for OR).

The 10-year rate of mortality for all patients in the cohort was 59\%. There were considerable differences between patients of different ages, however. The 10 -year mortality was $38 \%$ for patients aged under 70 years, and this increased to $80 \%$ for patients aged $80+$ years. The Cox regression model confirmed worse survival was associated with increasing age and the presence of any RCS comorbid condition (Table 2a), and to a lesser extent with patient sex. The model also suggested the overall long-term risk of death was higher among patients having EVAR than patients having open repair (adjusted hazard ratio $=1 \cdot 11,95 \%$ confidence interval (CI) $1 \cdot 07$ to $1 \cdot 16$ ). However, as in previous studies 8,9 , the pattern of survival after EVAR and OR was not consistent with the proportional hazards assumption of the standard Cox model, as illustrated in Figure 1 by the crossing of the Kaplan-Meier mortality curves around 18 months after surgery.

\section{Rates of AAA rupture after repair}

A total of 397 admissions for ruptured AAA (314 EVAR, 83 OR) were identified during the followup period, with the number rising steadily over time (Figure 2). At 5-years, $1.8 \%$ of patients who had an EVAR procedure were readmitted with a ruptured diagnosis compared with $0 \cdot 3 \%$ of patients who had OR (log-rank test, $\mathrm{p}<0 \cdot 001)$. At 9 years, the rupture rates for EVAR and OR were $3 \cdot 4$ and $0.9 \%$, respectively.

The Cox regression model for time from AAA repair to readmission with a ruptured aneurysm is shown in Table $2 b$. The results confirm a higher risk of rupture for EVAR patients compared to OR 
(hazard ratio $=3 \cdot 79,95 \%$ CI $2 \cdot 95$ to $4 \cdot 87$ ). The risk of rupture was only associated weakly with patient age and sex. In addition, although the risk of death on suffering a rupture was around $50 \%$ on average (Table 3), the low absolute rates of rupture for both procedures means that AAA ruptures represented a small proportion of all deaths.

\section{Patterns of long-term survival within patient groups}

To examine how survival after AAA repair was influenced by the patient characteristics, eight groups were defined by stratifying patients into four age categories ( $<70$ years, $70-74,75-79,80+$ years) and whether or not a patient had any of the RCS Charlson comorbid conditions. The patterns of 10-year mortality for the patient groups are described in Figure 3. The left-hand graphs contain patients who did not have any RCS Charlson comorbidity recorded while the right panel includes patients who did. The plots show estimated rates of mortality derived from the flexible parametric models (solid lines) alongside the Kaplan-Meier estimates (dashed lines) to demonstrate how well the models fit the data.

The graphs in Figure 3 reveal the heterogeneity in the survival trajectories for EVAR and OR across the patient groups, over both the short-term postoperative period (up to 12 months) and the subsequent 9-years of follow-up. For patients under 70 years with no RCS Charlson comorbidities, there was little difference in the mortality curves of the two procedures, and any short-term advantage for patients having EVAR was minimal. For both procedures, the expected survival over the 10 -year follow-up was around 100 months (Table 4). There was also little short-term advantage for EVAR among patients under 70 years with a Charlson comorbidity, but the survival trajectories begin to diverge after 24 months, such that the expected survival time over the 10 years for OR was 7.3 months greater than for EVAR (95\% CI $-9 \cdot 6$ to $-5 \cdot 0 ; \mathrm{p}<0.001)$. In the other six patient groups, OR was associated with a larger short-term risk of death compared with EVAR, with the absolute difference increasing among older patients and when patients had a Charlson comorbidity. For patients over 80 years with a comorbidity, the absolute difference in short-term survival offered by EVAR compared to OR was substantial (8.1\% for EVAR vs $18.3 \%$ for OR after 6 months). 
The graphs also show the different durations over which the early survival benefit of EVAR was maintained due to the different short-term and longer-term hazards for EVAR and OR. This resulted in EVAR having a small but statistically significant advantage over 10 years for patients aged 75-79 years with no Charlson comorbidity (difference $=3 \cdot 7,95 \%$ CI $0 \cdot 7$ to $6 \cdot 7 ; \mathrm{p}<0.001$ ) but the opposite was observed for patients aged $75-79$ with a comorbidity (difference $=-4 \cdot 5,95 \%$ CI $-7 \cdot 0$ to -2.0 ; $\mathrm{p}<0.001)$. While patients aged $80+$ years with a RCS comorbidity had the largest survival benefit from EVAR over the short-term, the longer-term hazard for EVAR was higher than for OR and, at 10years, the expected survival times were similar for both procedures (Table 4).

\section{DISCUSSION}

Information on survival after elective repair of unruptured AAA from randomised clinical trials suggest that, while EVAR has higher rates of survival than OR over the first 6 months, this benefit is lost over the longer term1-5. The trials have also reported increased rates of rupture among patients having EVAR, which has raised concerns about the durability of EVAR. However, the interpretation of this evidence has proven contentious and clinical guidelines contain conflicting recommendations about which technique is preferred. For example, in the UK, the draft guideline from NICE recommended open surgical repair for elective AAA, while the European Society for Vascular Surgery Guideline recommended EVAR 17,18 .

The results from this study extend the evidence base by revealing the variation in the survival trajectories after elective EVAR and OR. As expected, increasing age had a strong overall effect, with 10-year mortality being $38 \%$ for patients under 70 years but increasing to $80 \%$ for patients over 80 . But, while previous studies have reported that age and individual comorbidities influence long-term survival12, a key finding of this study is how these factors produce different patterns of short-term and long-term mortality, which combine to produce an array of survival trajectories for patients having EVAR and OR. For patients under 70 years with no RCS comorbidity, the trajectories for EVAR and OR were similar over the 10-year follow-up period. In contrast, for patients over 80 years with an 
RCS Charlson comorbidity, the absolute difference in short-term survival offered by EVAR compared to OR was substantial but after 10 years, the mortality rate for EVAR was higher compared to OR ( $87 \%$ vs $79 \%$ ). The study also found a higher risk of rupture among patients who had EVAR than OR (3.4\% vs $0.9 \%$ after 9 years), which was only weakly associated with patient factors. The low absolute rates of rupture for both procedures suggest AAA ruptures contributed only a small proportion of all the deaths after AAA repair.

The overall results from this study are consistent with the long-term outcomes reported by the RCTs, showing that the early survival advantage of EVAR was lost over a period of 1-3 years1-6. In addition, the observed rates of rupture after EVAR and OR are comparable to the results reported by Stather et al using pooled data from RCTs and cohort studies $(1.9 \%$ vs $0.3 \%, \mathrm{P}<0.001)$. Data from the OVER trial suggested survival might be better after EVAR among patients younger than 70 years but not among those over 705. This finding was not replicated in a meta-analysis of RCT data2, nor by a US population-based cohort study6. The latter demonstrated that the early advantage of EVAR was greater among patients with increasing age, but the comparative effectiveness of EVAR was only reported for the overall cohort and not for any patient subgroups. Desai et al reported gender differences in short and long-term survival following OR and EVAR, finding that women had a significantly worse long-term survival following OR 19 . This study also found differences between men and women but these were small compared to age and comorbidity.

Evidence on the impact of individual comorbidities on long-term survival after AAA repair was summarised in a recent systematic review12, but it did not describe how the survival trajectories might be affected, reporting the effects as hazard ratios. In pooled RCT data, Powell et al2 found weak evidence to suggest renal dysfunction and coronary artery disease might have a differential effect in the first 6 months, whereas peripheral artery disease might have an effect after 6 months. Populationbased cohort studies have so far not reported survival trajectories for AAA patients with different comorbidities6-9. 
The economic evaluation undertaken by NICE in developing its draft guideline included age, sex and aneurysm diameter in the cost-effectiveness model for elective AAA repair as modifiers of the probability of perioperative (30 day) and long-term mortality 17. The estimated net benefit of OR compared to EVAR reduced as patient age increased, but this was insufficient to change its baseline finding that OR was more cost-effective than EVAR. However, the results might be conservative due to the omission of comorbidities and because distinguishing only between peri-operative and longterm periods of survival will not capture the timeframe over which EVAR and OR show differential mortality rates.2,6 The adoption of a consistent set of time points for reporting long-term mortality (such as those recommended by the British Society of Endovascular Therapy 20 ) would help these limitations be avoided.

This study only stratified patients by two patient characteristics (age and the presence of comorbidity) and within each of the groups, there will be heterogeneity among the patients with regard to anatomical factors related to their AAA, the combinations of comorbid conditions and their overall physical fitness21, all of which will influence short- and long-term outcomes after aortic repair. In particular, there may be significant anatomical differences in aneurysm morphology such as a more hostile neck anatomy22 which can lead to OR being more appropriate. Among older patients, there may be a reluctance to perform OR in the light of the higher perioperative risk, and a willingness to compromise on anatomical suitability for EVAR to reduce this risk.

\section{Study strengths and limitations}

Our study has several strengths, in particular, the large number of EVAR and OR procedures. Hospital Episode Statistics captures all admissions to English NHS hospitals and therefore the cohort is a representative sample. Few elective AAA repairs are performed in independent hospitals. In addition, the linkage of patient records to the ONS death register means the study had complete follow-up information on all-cause mortality. Finally, the study used data on AAA repairs undertaken up until December 2015, which will reflect the use of the more recent EVAR devices as well as the increased popularity of EVAR procedures. 
The study has several limitations. First, there is the potential for inaccuracies in the coding of AAA diagnoses and procedures within $\mathrm{HES}_{23}$, errors which would affect the definition of the patient cohort. However, previous studies reported good consistency in the coding of AAA diagnoses and procedures24, finding HES to be $94.9 \%$ consistent when diagnoses and procedural coding combinations were examined. Consequently, bias from misclassifying EVAR and OR procedures is unlikely to affect the study results. Bias could arise from the incomplete or miscoding of the comorbid conditions included in the RCS Charlson Index 15 and data quality might have varied over time. The omission of codes is the more common error and would result in patients being wrongly included in the "no RCS comorbidity" subgroups. But any mis-classification bias is unlikely to change the study conclusions given the degree of heterogeneity in the long-term survival trajectories observed across all the subgroups.

Second, the HES database lacked information on various relevant clinical fields such as the size of the aneurysm and other anatomical details of the aorta, which are useful in determining the suitability of a patient for either procedure and for risk adjustment. It also lacked information on the EVAR device used. There are no data on other known prognostic risk factors including various physiological variables (e.g. creatinine) or assessments of fitness like the American Society of Anesthesiologists (ASA) grade. This restricted our ability to explore differences between patient groups extensively. This could be possible using records from a national, population-based vascular registry if long-term follow-up data were available alongside the details of the AAA repair. As the linkage of healthcare datasets with other national datasets become more feasible, studies that provide greater insight into the survival trajectories for different patients after EVAR and OR will hopefully be forthcoming.

In summary, this study highlights the considerable variation in long-term survival patterns after elective OR and EVAR for unruptured AAA across patient groups. This variation arose from changes in the short-term and long-term mortality rates associated with patients of different age and other serious comorbid conditions. The different profiles of risks and benefits over time has important 
implications for clinicians and patients, and the appropriate treatment will depend upon the value given to avoiding a poor outcome in the immediate future compared with a potentially longer life expectancy25. This has important implications for recommendations in clinical guidelines, suggesting a simple recommendation in favour of either OR or EVAR ignores the fact that individuals will face different trade-offs when making treatment decisions26. Moreover, these trade-offs will be valued differently by patients and surgeons 27 and it is important that clinicians can provide patients with appropriate information so that they can make an informed decisions about their preferred intervention. 


\section{Acknowledgments}

Hospital Episode Statistics (HES) were made available by NHS Digital (Copyright 2017, Re-used with the permission of NHS Digital. All rights reserved.) Approvals for the use of anonymised HES data were obtained as part of the standard approval process. The authors thank Lynn Copley for providing the required extract from the HES database.

No preregistration exists for the reported studies reported in this article.

\section{Abbreviations}

AAA, abdominal aortic aneurysm; OR, open repair; EVAR, endovascular repair; RMST, restricted mean survival time; RCT, randomised controlled trial; HES, Hospital Episode Statistics; ICD-10, International Classification of Diseases, 10th revision; OPCS, UK Office for Population Censuses and Surveys classification, 4 th revision. 


\section{References}

1. Stather PW, Sidloff D, Dattani N, Choke E, Bown MJ, Sayers RD. Systematic review and metaanalysis of the early and late outcomes of open and endovascular repair of abdominal aortic aneurysm. Br J Surg. 2013; 100: 863-872.

2. Powell JT, Sweeting MJ, Ulug P, Blankensteijn JD, Lederle FA, Becquemin JP, Greenhalgh RM, on behalf of the EVAR-1, DREAM, OVER and ACE Trialists. Meta-analysis of individualpatient data from EVAR-1, DREAM, OVER and ACE trials comparing outcomes of endovascular or open repair for abdominal aortic aneurysm over 5 years. Br J Surg. 2017; 104: $166-178$.

3. De Bruin JL, Baas AF, Buth J, Prinssen M, Verhoeven EL, Cuypers PW, van Sambeek MR, Balm R, Grobbee DE, Blankensteijn JD, DREAM Study Group. Long-term outcome of open or endovascular repair of abdominal aortic aneurysm. N Engl J Med 2010; 362: 1881-1889.

4. Patel R, Sweeting MJ, Powell JT, Greenhalgh RM. Endovascular versus open repair of abdominal aortic aneurysm in 15-years' follow-up of the UK endovascular aneurysm repair trial 1 (EVAR trial 1): a randomised controlled trial. United Kingdom EVAR Trial Investigators. Lancet 2016; 388: 2366-2374.

5. Lederle FA, Freischlag JA, Kyriakides TC, Matsumura JS, Padberg FT Jr, Kohler TR, Kougias P, Jean-Claude JM, Cikrit DF, Swanson KM, Group Ovacs. Long-term comparison of endovascular and OR of abdominal aortic aneurysm. N Engl J Med 2012; 367: 1988-1997.

6. Schermerhorn ML, Buck DB, O’Malley AJ, Curran T, McCallum JC, Darling J, Landon BE. Long-term outcomes of abdominal aortic aneurysm in the medicare population. $N$ Engl J Med 2015; 373: 328-338.

7. Jackson RS, Chang DC, Freischlag JA. Comparison of long-term survival after open vs endovascular repair of intact abdominal aortic aneurysm among Medicare beneficiaries. JAMA 2012; 307: 1621-1628.

8. Wahlgren CM, Malmstedt J, on behalf of the Swedish Vascular Registry. Outcomes of endovascular abdominal aortic aneurysm repair compared with open surgical repair in high-risk patients: Results from the Swedish Vascular Registry. J Vasc Surg 2008; 48: 1382-1389. 
9. Chang DC, Parina RP, Wilson SE. Survival After Endovascular vs Open Aortic Aneurysm Repairs. JAMA Surg 2015; 150(12): 1160-1166.

10.Sorensen HT, Lash TL, Rothman KJ. Beyond randomized controlled trials: a critical comparison of trials with nonrandomised studies. Hepatology 2006; 44: 1075-1082.

11.Hopkins R, Bowen J, Campbell K, et al. Effects of study design and trends for EVAR versus OSR. Vasc Health Risk Manag. 2008;4(5):1011-1022

12.Khashram M, Williman JA, Hider PN, Jones GT, Roake JA. Systematic review and metaanalysis of factors influencing survival following abdominal aortic aneurysm repair. Eur J Vasc Endovasc Surg. 2016; 51(2): 203-15.

13. Royston P, Lambert PC. Flexible Parametric Survival Analysis Using Stata: Beyond the Cox Model. College Station: StataCorp LP, 2011.

14. Royston P, Parmar MK. Restricted mean survival time: an alternative to the hazard ratio for the design and analysis of randomized trials with a time-to-event outcome. BMC Med Res Methodol 2013; 13: 152.

15.Armitage JN, van der Meulen JH. Identifying co-morbidity in surgical patients using administrative data with the Royal College of Surgeons Charlson score. Br J Surg 2010; 97: 772781.

16.Bradburn MJ, Clark TG, Love SB, Altman DG. Survival Analysis Part III: Multivariate data analysis - choosing a model and assessing its adequacy and fit. Br J Cancer 2003; 89: 605-611.

17.Abdominal aortic aneurysm: diagnosis and management - NICE. guideline DRAFT (May 2018). https://www.nice.org.uk/guidance/gid-cgwave0769/documents/short-version-of-draft-guideline. (accessed 15th January 2019).

18.European Society for Vascular Surgery Guidelines. Management of Abdominal Aorto-iliac Artery Aneurysms (January 2019). http://www.esvs.org/journal/guidelines/. (accessed 15th January 2019).

19.Desai M, Choke E, Sayers RD, Nath M, Bown MJ. Sex-related trends in mortality after elective abdominal aortic aneurysm surgery between 2002 and 2013 at National Health Service hospitals in England: less benefit for women compared with men. Eur Heart J 2016; 37, 3452-3460. 
20.Boyle JR, Thompson MM, Vallabhaneni SR, Bell RE, Brennan JA, Browne TF, Cheshire NJ, Hinchliffe RJ, et al Pragmatic minimum reporting standards for endovascular abdominal aortic aneurysm repair. J Endovasc Ther. 2011; 18(3): 263-71.

21.Arya S, Kim SI, Duwayri Y, Brewster LP, Veeraswamy R, Salam A, Dodson TF. Frailty increases the risk of 30-day mortality, morbidity, and failure to rescue after elective abdominal aortic aneurysm repair independent of age and comorbidities. J Vasc Surg 2015; 61(2):324-331.

22. Antoniou GA, Georgiadis GS, Antoniou SA, Kuhan G, Murray D. A meta-analysis of outcomes of endovascular abdominal aortic aneurysm repair in patients with hostile and friendly neck anatomy. J Vasc Surg 2013; 57(2):527-38.

23.NHS Digital. Data Quality Maturity Index. Introduction and Methodology. 8 November 2016; http://content.digital.nhs.uk/media/22883/DQMI-3-Methodology/pdf/DQMI3_Methodology.pdf.

24.Johal A, Mitchell D, Lees T, Cromwell D, van der Meulen J. Use of Hospital Episode Statistics to investigate abdominal aortic aneurysm surgery. Br J Surg 2012; 99(1): 66-72.

25.Rakow T. Differences in belief about likely outcomes account for differences in doctors' treatment preferences: but what accounts for the differences in belief? Qual Health Care. 2001; 10 Suppl 1: i44-9.

26. Reise JA, Sheldon H, Earnshaw J, Naylor AR, Dick F, Powell JT et al. Patient preference for surgical method of abdominal aortic aneurysm repair: postal survey. Eur J Vasc Endovasc Surg 2010; 39: 55-61.

27.Kipp R, Lehman J, Israel J, Edwards N, Becker T, Raval AN. Patient preferences for coronary artery bypass graft surgery or percutaneous intervention in multivessel coronary artery disease. Catheter Cardiovasc Interv. 2013; 82: 212-218. 
Table 1. Patient characteristics at time of procedure among elective AAA repairs performed between 2006 and 2015 , by procedure type.

\begin{tabular}{|c|c|c|c|c|c|}
\hline & OR & $\%$ & EVAR & $\%$ & Total \\
\hline \multicolumn{6}{|c|}{ Age group (years) } \\
\hline Under 70 & 5676 & $36 \cdot 6$ & 4428 & $20 \cdot 5$ & 10104 \\
\hline 70 to 74 & 3889 & $25 \cdot 1$ & 4554 & $21 \cdot 1$ & 8443 \\
\hline 75 to 79 & 3694 & $23 \cdot 8$ & 5933 & $27 \cdot 4$ & 9627 \\
\hline $80 \&$ over & 2264 & $14 \cdot 6$ & 6700 & $31 \cdot 0$ & 8964 \\
\hline \multicolumn{6}{|l|}{ Sex } \\
\hline Male & 13388 & $86 \cdot 2$ & 19212 & $88 \cdot 9$ & 32600 \\
\hline Female & 2135 & $13 \cdot 8$ & 2403 & $11 \cdot 1$ & 4538 \\
\hline \multicolumn{6}{|c|}{ No. of comorbidities (Charlson score) } \\
\hline 0 & 6270 & $40 \cdot 4$ & 6853 & $31 \cdot 7$ & 13123 \\
\hline 1 & 5576 & $35 \cdot 9$ & 7497 & $34 \cdot 7$ & 13073 \\
\hline 2 or more & 3677 & $23 \cdot 7$ & 7265 & $33 \cdot 6$ & 10942 \\
\hline Total & 15523 & & 21615 & & 37138 \\
\hline
\end{tabular}


Table 2a. Results of Cox regression model for survival time of all patients undergoing elective AAA repair $(n=37$ 138).

\begin{tabular}{lccc}
\hline & Hazard Ratio & 95\% Confidence interval & P value \\
\hline Open repair & 1 & 1.07 to 1.16 & $<0.001$ \\
EVAR & 1.11 & & \\
& & & \\
Age group (years) & 1 & 1.37 to 1.54 & $<0.001$ \\
$\quad$ Under 70 & 1.45 & 1.95 to 2.18 & $<0.001$ \\
70 to 74 & 2.06 & 2.95 to 3.29 & $<0.001$ \\
75 to 79 & 3.11 & & \\
80 or more & 1 & 1.03 to 1.14 & 0.001 \\
Male & 1.09 & & \\
Female & & & \\
RCS Charlson Comorbidity Score & 1 & 1.65 to 1.79 & $<0.001$ \\
$\quad 0$ & 1.72 & & \\
1 or more & & & \\
\hline
\end{tabular}

Table $2 \mathrm{~b}$. Results of Cox regression model for time to readmission with a ruptured diagnosis.

\begin{tabular}{lccc}
\hline & Hazard Ratio & 95\% Confidence interval & P value \\
\hline $\begin{array}{l}\text { Open repair } \\
\text { EVAR }\end{array}$ & 1 & 2.95 to 4.87 & $<0.001$ \\
& & & \\
Age group (years) & 1 & & \\
$\quad$ Under 70 & 0.87 & 0.62 to 1.20 & 0.384 \\
$70-74$ & 1.28 & 0.96 to 1.70 & 0.095 \\
$75-79$ & 1.43 & 1.08 to 1.90 & 0.013 \\
$\quad 80$ or more & 1 & & \\
Male & 1.37 & 1.05 to 1.79 & 0.022 \\
Female & & & \\
RCS Charlson Comorbidity Score & 1 & & \\
$\quad 0$ & 1.16 & 0.94 to 1.44 & \\
1 or more & & & \\
\hline
\end{tabular}


Table 3. Readmission and death by ruptured aneurysm. Numbers and percentage of patients readmitted with a ruptured diagnosis and percentage experiencing death from ruptured aneurysm within 5 years following an initial elective EVAR procedure.

\begin{tabular}{|c|c|c|c|}
\hline Patient group & $\mathrm{N}$ & $\begin{array}{c}\% \text { ruptured readmission at } 5 \\
\text { years }(95 \% \mathrm{CI})\end{array}$ & $\begin{array}{l}\% \text { ruptured deaths at } 5 \text { years } \\
\qquad(95 \% \mathrm{CI})\end{array}$ \\
\hline \multicolumn{4}{|c|}{ No RCS Charlson comorbidities } \\
\hline Under 70 yrs & 1413 & $1 \cdot 3(0 \cdot 7,2 \cdot 3)$ & $0 \cdot 2(0 \cdot 1,0 \cdot 7)$ \\
\hline $70-74$ yrs & 1384 & $0.9(0 \cdot 4,1 \cdot 8)$ & $0.4(0 \cdot 2,1 \cdot 1)$ \\
\hline $75-79$ yrs & 1801 & $1 \cdot 5(1 \cdot 0,2 \cdot 4)$ & $1 \cdot 2(0 \cdot 7,1 \cdot 9)$ \\
\hline 80 yrs \& over & 2255 & $2 \cdot 2(1 \cdot 5,3 \cdot 1)$ & $1 \cdot 2(0 \cdot 8,1 \cdot 8)$ \\
\hline Total & 6853 & $1 \cdot 6(1 \cdot 2,2 \cdot 0)$ & $0 \cdot 8(0 \cdot 6,1 \cdot 1)$ \\
\hline \multicolumn{4}{|c|}{ One or more RCS Charlson comorbidities } \\
\hline Under 70 yrs & 3015 & $1 \cdot 7(1 \cdot 2,2 \cdot 4)$ & $0 \cdot 7(0 \cdot 4,1 \cdot 1)$ \\
\hline $70-74$ yrs & 3170 & $1 \cdot 7(1 \cdot 2,2 \cdot 4)$ & $0.7(0 \cdot 4,1 \cdot 2)$ \\
\hline $75-79$ yrs & 4132 & $1 \cdot 9(1 \cdot 4,2 \cdot 5)$ & $0 \cdot 8(0 \cdot 6,1 \cdot 2)$ \\
\hline 80 yrs \& over & 4445 & $2 \cdot 3(1 \cdot 8,2 \cdot 9)$ & $1 \cdot 5(1 \cdot 1,2 \cdot 0)$ \\
\hline Total & 14762 & $1 \cdot 9(1 \cdot 7,2 \cdot 3)$ & $1 \cdot 0(0 \cdot 8,1 \cdot 2)$ \\
\hline
\end{tabular}


Table 4. Restricted mean survival times (RMST) in months for OR and EVAR procedures over the 10-year follow-up period, by patient group.

\begin{tabular}{|c|c|c|c|c|c|c|}
\hline Patient group & $\begin{array}{l}\text { RMST for } \\
\text { OR }\end{array}$ & $\begin{array}{l}\text { Std } \\
\text { Error }\end{array}$ & $\begin{array}{c}\text { RMST for } \\
\text { EVAR }\end{array}$ & $\begin{array}{c}\text { Std } \\
\text { Error }\end{array}$ & Difference & $95 \% \mathrm{CI}$ \\
\hline \multicolumn{7}{|c|}{ No RCS Charlson comorbidities } \\
\hline Under 70 yrs & $105 \cdot 2$ & 0.7 & $104 \cdot 3$ & $1 \cdot 0$ & $-1 \cdot 0$ & $-3 \cdot 4$ to $1 \cdot 5$ \\
\hline 70 to $74 \mathrm{yrs}$ & $98 \cdot 6$ & $1 \cdot 0$ & $99 \cdot 1$ & $1 \cdot 1$ & $0 \cdot 5$ & $-2 \cdot 4$ to $3 \cdot 4$ \\
\hline 75 to $79 \mathrm{yrs}$ & $87 \cdot 8$ & $1 \cdot 1$ & $91 \cdot 5$ & $1 \cdot 1$ & $3 \cdot 7$ & 0.7 to $6 \cdot 7$ \\
\hline 80 yrs \& over & $75 \cdot 3$ & $1 \cdot 5$ & $73 \cdot 4$ & $1 \cdot 0$ & $-1 \cdot 9$ & $-5 \cdot 4$ to $1 \cdot 6$ \\
\hline \multicolumn{7}{|c|}{ One or more RCS Charlson comorbidities } \\
\hline Under 70 yrs & $96 \cdot 2$ & $0 \cdot 8$ & $88 \cdot 9$ & $0 \cdot 9$ & $-7 \cdot 3$ & $-9 \cdot 6$ to $-5 \cdot 0^{*}$ \\
\hline 70 to $74 \mathrm{yrs}$ & $84 \cdot 2$ & $0 \cdot 9$ & $82 \cdot 1$ & $0 \cdot 9$ & $-2 \cdot 1$ & $-4 \cdot 7$ to $0 \cdot 5$ \\
\hline 75 to 79 yrs & $75 \cdot 8$ & $1 \cdot 0$ & $71 \cdot 3$ & $0 \cdot 8$ & $-4 \cdot 5$ & $-7 \cdot 0$ to $-2 \cdot 0^{*}$ \\
\hline 80 yrs \& over & $62 \cdot 1$ & $1 \cdot 3$ & $59 \cdot 7$ & $0 \cdot 7$ & $-2 \cdot 4$ & $-5 \cdot 3$ to 0.5 \\
\hline
\end{tabular}


Figure 1. Unadjusted Kaplan-Meier failure estimates for patients undergoing elective OR and EVAR for unruptured AAA.

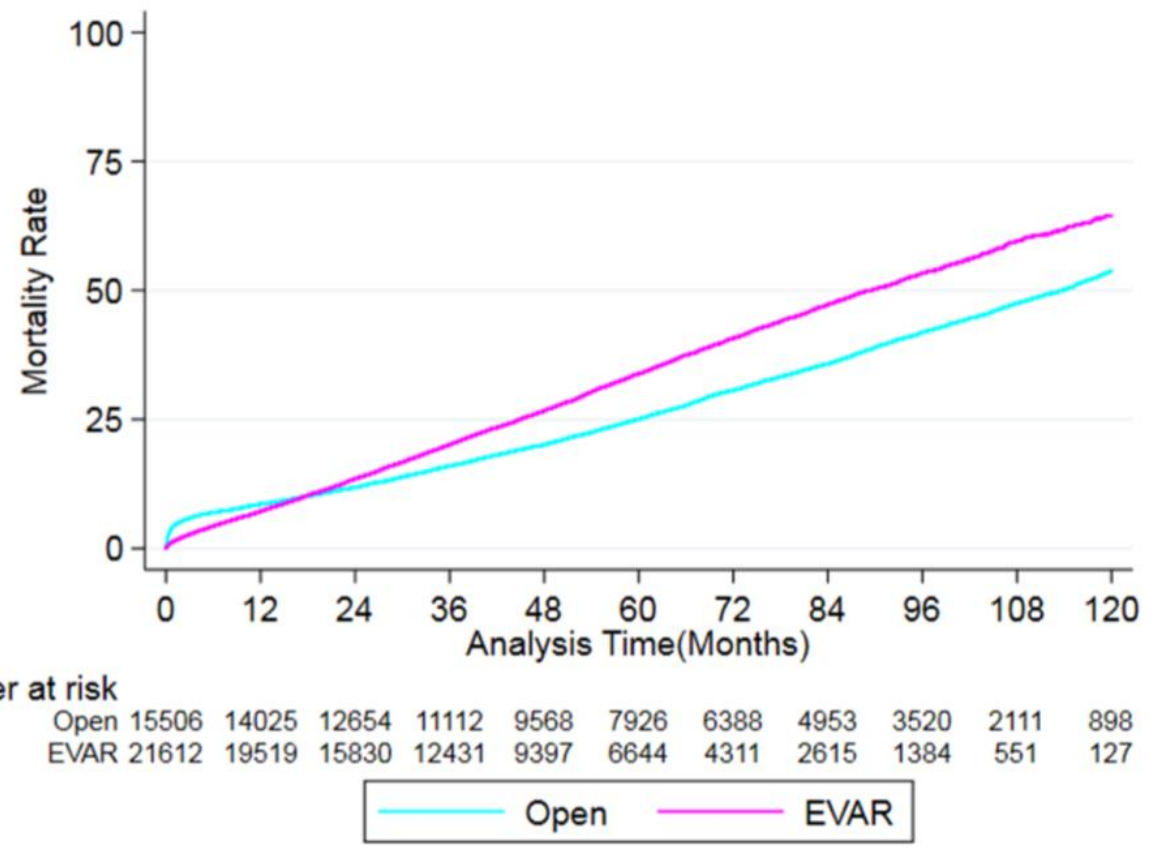


Figure 2. Time to rupture cumulative incidence curves for all patients. Outcomes for patients having EVAR and ORs are indicated in red and blue, respectively.

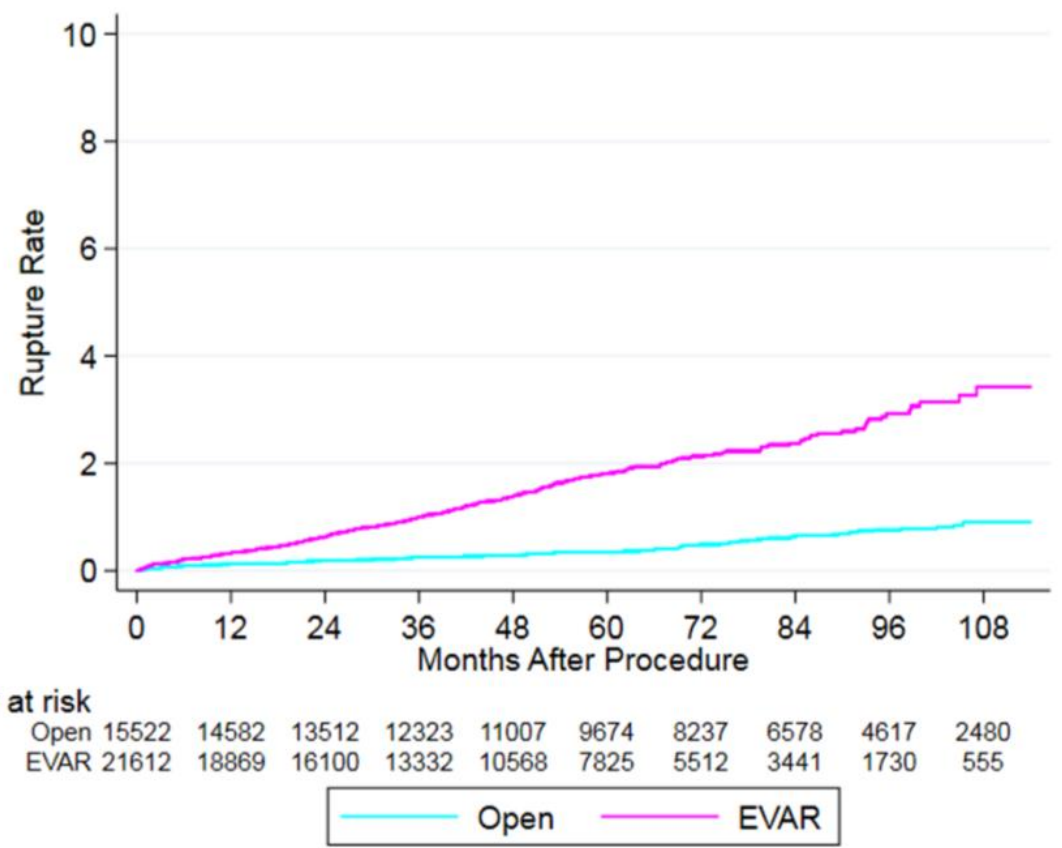


Figure 3. Mortality curves for patients stratified by age. Panels on the left shows patients who did not have a comorbidity while panels on the right show patients who did. Outcomes for patients having EVAR and ORs are indicated in red and blue, respectively. Dashed and solid lines show curves estimated from Kaplan-Meier and flexible parametric model, respectively.
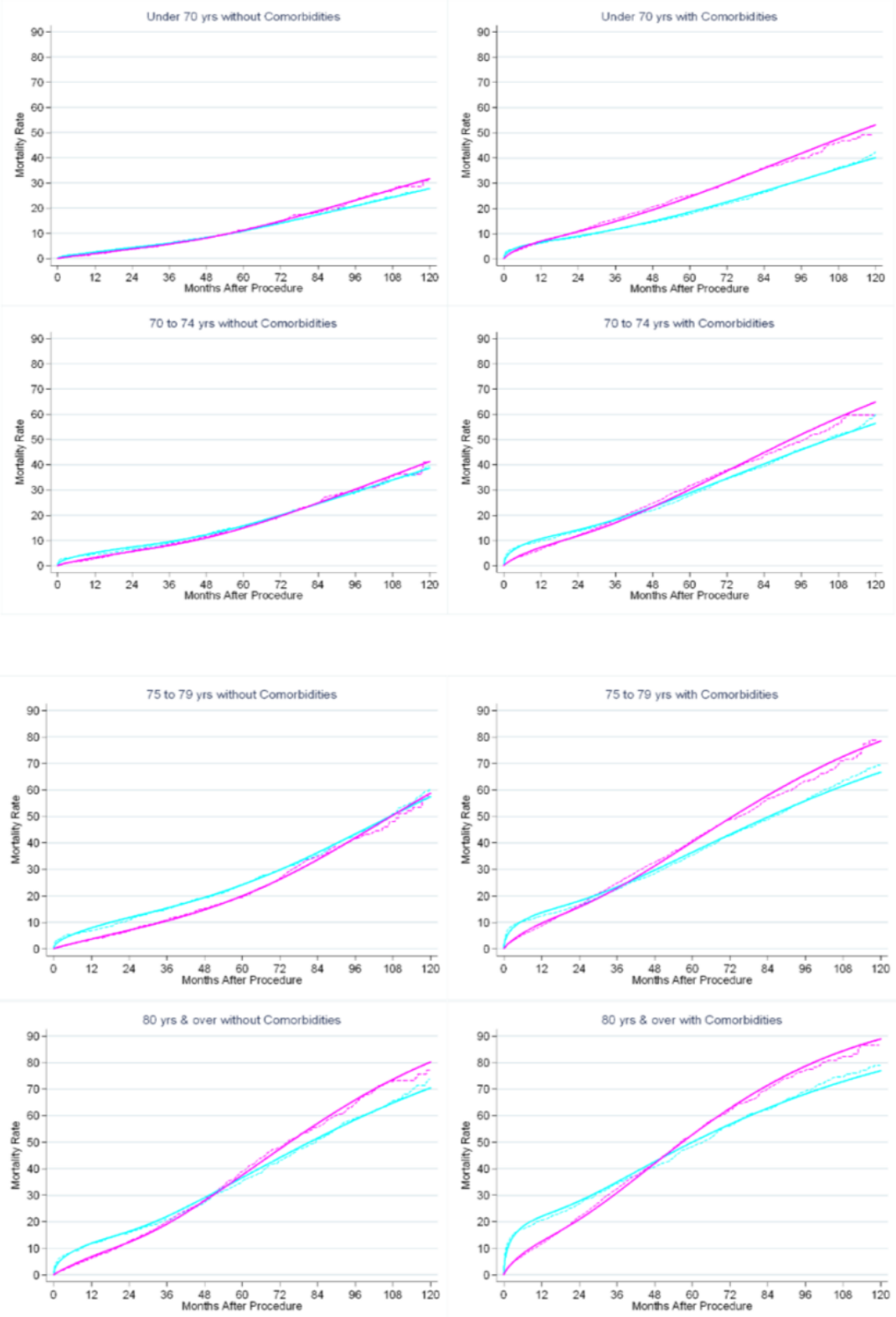


\section{Supplementary}

Table S1: Results of Cox regression model for all patients undergoing AAA repair $(n=37,138)$

\begin{tabular}{|c|c|c|c|}
\hline & Hazard Ratio & 95\% Confidence interval & $\mathrm{P}$ value \\
\hline OR & $1 \cdot 00$ & & $<0 \cdot 001$ \\
\hline EVAR & $1 \cdot 11$ & 1.07 to $1 \cdot 16$ & \\
\hline Age Group (years) & & & $<0 \cdot 001$ \\
\hline Under 70 & $1 \cdot 00$ & & \\
\hline 70 to 74 & 1.45 & 1.37 to 1.54 & \\
\hline 75 to 79 & $2 \cdot 06$ & 1.95 to $2 \cdot 18$ & \\
\hline 80 or more & $3 \cdot 11$ & $2 \cdot 95$ to $3 \cdot 29$ & \\
\hline Male & $1 \cdot 00$ & & $0 \cdot 001$ \\
\hline Female & $1 \cdot 09$ & 1.03 to $1 \cdot 14$ & \\
\hline \multicolumn{4}{|c|}{ Charlson Comorbidity Index } \\
\hline 0 & $1 \cdot 00$ & & $<0 \cdot 001$ \\
\hline 1 or more & 1.72 & 1.65 to 1.79 & \\
\hline
\end{tabular}


Table S2:- Results of the Schoenfeld residual test

\begin{tabular}{|c|c|c|c|c|}
\hline & $\rho$ & Chi-square 2 & $\begin{array}{l}\text { Degrees of } \\
\text { freedom }\end{array}$ & Prob $>\chi^{2}$ \\
\hline EVAR & $0 \cdot 103$ & $134 \cdot 62$ & 1 & $<0 \cdot 001$ \\
\hline Age at procedure & & & 1 & \\
\hline Under 70 & & & & \\
\hline 70 to 74 & $0 \cdot 011$ & 1.40 & 1 & $0 \cdot 237$ \\
\hline 75 to 79 & $0 \cdot 026$ & $8 \cdot 72$ & 1 & 0.003 \\
\hline 80 or more & $0 \cdot 027$ & $9 \cdot 47$ & 1 & $0 \cdot 002$ \\
\hline Gender & $0 \cdot 008$ & $0 \cdot 86$ & 1 & $0 \cdot 353$ \\
\hline Comorbidity $\geq 1$ & $-0 \cdot 070$ & $59 \cdot 90$ & 1 & $<0.001$ \\
\hline Global test & & $221 \cdot 01$ & 6 & $<0 \cdot 001$ \\
\hline
\end{tabular}

$P$ values $<0.05$ indicate violation of proportional hazard assumption. 
Figure S1: Log-log plot by procedure. Non-parallel lines indicate non-proportional hazards.

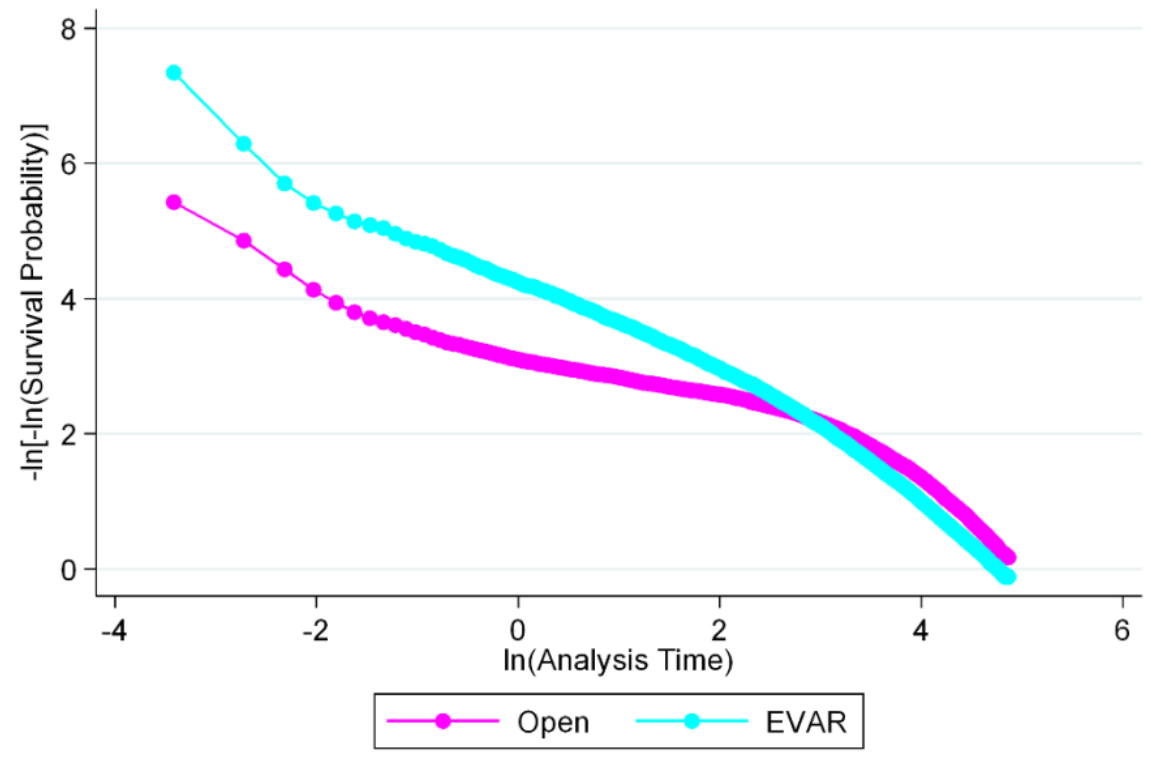

